\title{
High Resolution BPM for Linear Colliders
}

\author{
C. Simon", S. Chel*, M. Luong*, O. Napoly*, J. Novo*, D. Roudier*, \\ N. Baboi" ${ }^{\#}$ D. Noelle ${ }^{\#}$, N. Mildner ${ }^{\#}$ K. Zapfe ${ }^{\#}$ and N. Rouvière ${ }^{\forall}$ \\ ${ }^{*}$ Centre à l'Energie Atomique/Saclay, DSM/DAPNIA/SACM, F91191 Gif-sur-Yvette Cedex France \\ ${ }^{\#}$ Deutsches Elektronen Synchroton, Notkestasse 85, D-22603 Hamburg, Germany \\ ${ }^{\forall}$ Centre National de Recherche Scientifique, IN2P3 - IPN/Orsay, F91406 Orsay Cedex France
}

\begin{abstract}
A high resolution Beam Position Monitor (BPM) is necessary for the beam-based alignment systems of high energy and low emittance electron linacs. Such a monitor is developed in the framework of the European CARE/SRF programme, in a close collaboration between DESY and CEA/DSM/DAPNIA. This monitor is a radiofrequency re-entrant cavity, which can be used either at room or cryogenic temperature, in an environment where dust particle contamination has to be avoided, such as superconducting cavities in a cryomodule. A first prototype of a re-entrant BPM has already delivered measurements at $2 \mathrm{~K}$ inside the first cryomodule (ACC1) on the TESLA Test Facility 2 (TTF2). The performances of this BPM are analyzed both experimentally and theoretically, and the limitations of this existing system clearly identified. A new cavity and new electronics have been designed in order to improve the position resolution down to $1 \mu \mathrm{m}$ and the damping time down to $10 \mathrm{~ns}$.
\end{abstract}

Keywords: Beam position monitor, High resolution, RF cavity, Calibration, Signal processing. PACS: $29.27 . \mathrm{Eg}$

\section{INTRODUCTION}

This paper presents the evolution of a re-entrant beam position monitor (BPM), developed by the CEA Saclay in collaboration with DESY, in the framework of the CARE/SRF European programme. An existing re-entrant BPM is installed at cryogenic temperature inside the cryomodule (ACC1) on the TESLA Test Facility 2 (TTF2). The position and time resolution, which are experimentally and theoretically analyzed, show the limitations of this system. Due to the bandpass filter bandwidth, which is only $8 \mathrm{MHz}$, the bunch to bunch measurement on TTF2 and XFEL is impossible for this BPM system. To achieve a time resolution about $100 \mathrm{~ns}$ for single bunch and multi bunch operations, the bandpass filter bandwidth has to be modified. In this paper, a comparison between three solutions in terms of resolutions, noise and output signal levels accounting for the cavity radiofrequency characteristics and the signal processing, is presented. Finally, the solution which requires a new design for both the BPM cavity and the processing electronics is adopted. With this new BPM, the position resolution is expected to be better than $1 \mu \mathrm{m}$ and the time resolution is high enough to ensure bunch to bunch measurement for XFEL or ILC. 


\section{BPM INSTALLED ON TTF2 WITH 8 MHZ BANDWIDTH}

\section{RF Characteristics and Signal Processing}

A re-entrant BPM consists of a coaxial cavity arranged around the beam pipe [1]. It has already proved its capability to be operated at cryogenic temperature inside the cryomodule (ACC1).

Passing through this cavity, the beam excites electromagnetic fields (resonant modes), which are coupled by four feedthroughs to the outside. The monopole mode signal is proportional to beam intensity and does not depend on the beam position contrary to the dipole mode signal which is additionally proportional to the distance of the beam from the centre axis of the monitor. The fixing of the antenna tips to the inner part of the cavity strongly over coupled the cavity [2].

To produce a model of the system and simulate its performance, the RF characteristics of this cavity (eigenmodes frequencies, coupling and longitudinal R/Q) are calculated with the HFSS code provided by Ansoft Corporation. The results are given in the Table 1.

TABLE 1. RF characteristics of the BPM installed in ACC1.

\begin{tabular}{|l|c|c|c|c|}
\hline Eigen modes & $\mathbf{F}(\mathbf{G H z})$ & $\mathbf{Q}_{\text {ext }}$ & $\mathbf{R} / \mathbf{Q}_{\mathbf{I}}(\mathbf{\Omega}) \mathbf{a t} \mathbf{5} \mathbf{~ m m}$ & $\mathbf{R} / \mathbf{Q}_{\mathbf{I}}(\mathbf{\Omega}) \mathbf{a t} \mathbf{1 0} \mathbf{~ m m}$ \\
\hline Monopole mode & 1.58 & 2.15 & 20.2 & 20.4 \\
\hline Dipole mode & 2.01 & 4.11 & 0.53 & 2.20 \\
\hline Quadrupole mode & 2.25 & 0.97 & 0.01 & 0.01 \\
\hline Parasitic dipole mode & 2.30 & 1.00 & 0.30 & 1.00 \\
\hline Parasitic monopole mode & 2.34 & 1.02 & 3.70 & 4.10 \\
\hline
\end{tabular}

Due to the low external $\mathrm{Q}$, the single bunch response of the cavity has to be broadened before its acquisition. This is done with a bandpass Bessel filter. The center frequency is chosen at $650 \mathrm{MHz}$ as a multiple of the repetition bunch frequency $(216.66 \mathrm{MHz})$ on the first TTF injector. The $8 \mathrm{MHz}$ bandwidth of this filter defines the bandwidth of the system and determines the time resolution [3].

\section{Calibration Methods and Results}

A calibration was carried out on the BPM. The TTF2 was operated in single bunch for these measurements which produced statistics and correlations. Two methods were used to calibrate this BPM called 9ACC1.

In the first method, the relative beam displacement at the BPM location was calculated in using a transfer matrix between steerers and BPM (made of drifts and accelerating cavities) for different values of drive current in the steerers:

$$
\Delta x=R_{12} * \Delta x^{\prime} \text { (where } \Delta \mathrm{x}^{\prime} \text { is the beam angle at steerer). }
$$

The HOM (Higher Order Mode) signals from cavity 8, the closest to 9ACC1, were minimized thanks to beam steering. This minimization gave an estimation of the BPM center. The beam tilt was neglected due to the closeness of BPM 9ACC1 to cavity 8 . An offset on $\Delta \mathrm{x}$ was added in the acquisition software to read a zero in this condition. Another calibration coefficient was computed from a linear fit of the predicted position to the measured position. 
The second method used three BPMs. The absolute beam position at the reentrant BPM (9ACC1) was calculated by extrapolation from the two other BPMs. All quadrupoles between the three BPMs were switched off to reduce errors and simplify calculation.

With the first calibration method (transfer matrix), 9ACC1 showed a linear range about $\pm 1.5 \mathrm{~mm}$ before saturation due to amplifiers or analog to digital converters, contrary to the second method which showed an unexpected non-linearity in the same range. The problem may arise from the long distance $(\sim 6 \mathrm{~m})$ between the first BPM and 9ACC1. On the Fig. 1 and Fig. 2, the plots of the position times the bunch charge read by $9 \mathrm{ACC} 1$ vs. the same quantity predicted by the first method are displayed for channels $\mathrm{X}$ and $\mathrm{Y}$. The nominal bunch charge was about $1 \mathrm{nC}$.
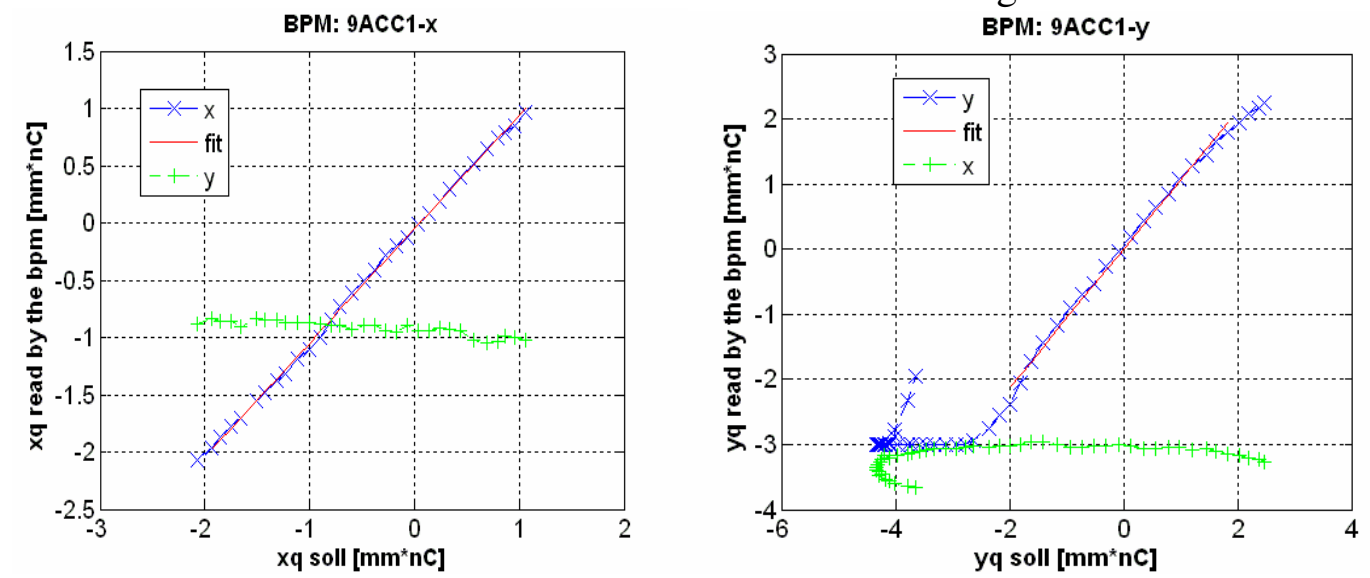

FIGURE 1 \& 2. Linear range about $\pm 1.5 \mathrm{~mm}$ of the BPM 9ACC1 installed on TFF2

The raw RMS resolution of the system directly measured by the standard deviation of the readings from 9ACC1 is around $50 \mu \mathrm{m}$ on the $\mathrm{X}$ channel. In using the correlation between different BPMs, the beam jitter can be canceled and the position resolution can be estimated around $20 \mu \mathrm{m}$. On the $\mathrm{Y}$ channel, the resolution is around $30 \mu \mathrm{m}$ after the beam jitter cancellation and around $70 \mu \mathrm{m}$ as a raw measurement.

These results show the limitation of the system. A new design is therefore necessary to achieve a position resolution better than 10 or even $1 \mu \mathrm{m}$ required by the XFEL or ILC linear accelerator.

\section{DESIGN OF THE MODIFIED BPM}

\section{RF Characteristics of the Cavity BPM}

To improve the time and position resolution of 9ACC1, a new BPM system (NBPM), based on the principle of a re-entrant cavity, was designed.

The signal voltage of the BPM is defined by the beam energy loss to the resonant modes and by the external coupling to the coaxial cables. In order to get a higher $\mathrm{Q}$ and therefore a longer signal in time without the need of a Bessel filter for signal shaping, the feedthroughs are moved $31.5 \mathrm{~mm}$ in the re-entrant part (Fig. 3). This 
antenna position also increases the separation of the RF modes (monopole and dipole mode) and facilitates the calibration. In the past, a critical point concerned the feedthrough fragility; $50 \%$ of the feedthroughs for 9ACC1 had to be rejected. With this new design, the machining of feedthroughs is simpler and the final product more robust. Several cryogenic and vacuum tests were applied to five new feedthroughs and all of them passed the tests successfully.

The BPM cavity is fabricated with stainless steel as compact as possible: $170 \mathrm{~mm}$ in length, $78 \mathrm{~mm}$ for the beam pipe and $152 \mathrm{~mm}$ in diameter as shown Fig. 3. The length of the cavity is minimized to satisfy the constraints imposed by the cryomodule. Twelve holes of $5 \mathrm{~mm}$ diameter are drilled at the end of the re-entrant part for more effective cleaning.
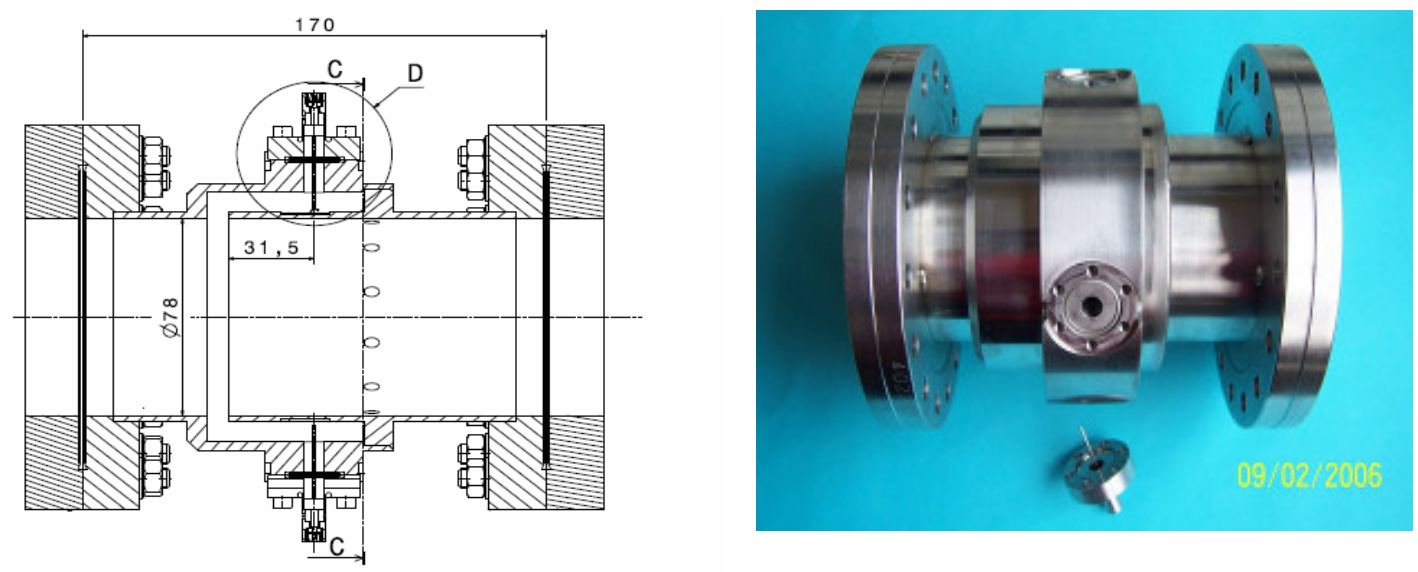

FIGURE 3. Drawing and photo of the modified BPM cavity.

The antennas fulfil the conditions of Ultra High Vaccum (UHV). They are assembled to the cavity by a conflat gasket. For each antenna, a CuBe RF contact is welded in the inner cylinder of the cavity to ensure electrical conduction between the feedthrough inner conductor and the cavity, providing a magnetic coupling loop. In order to avoid hydrogen out gassing on site, a heat treatment at $280{ }^{\circ} \mathrm{C}$ for 15 days is applied to the BPM cavity body instead of the usual treatment $\left(950{ }^{\circ} \mathrm{C}\right.$ for $\left.2 \mathrm{~h}\right)$ which may have drastically reduced the RF contact elasticity.

The resonant cavity is, first, simulated with the software HFSS (Ansoft) to determine its frequencies and coupling. The RF measurements, presented in Table 2, compare some computed quantities to measured values.

TABLE 2. RF characteristics of the NBPM.

\begin{tabular}{|c|c|c|c|c|c|c|}
\hline \multirow[t]{2}{*}{ Eigen modes } & \multicolumn{2}{|c|}{ F (GHz) } & \multicolumn{2}{|c|}{$\overline{\mathbf{Q}_{\text {ext }}}$} & \multirow{2}{*}{$\begin{array}{l}R / Q_{1}(\Omega) \\
\text { at } 5 \mathrm{~mm}\end{array}$} & \multirow{2}{*}{$\begin{array}{l}R / Q_{1}(\Omega) \\
\text { at } 10 \mathrm{~mm}\end{array}$} \\
\hline & calculated & measured & calculated & measured & & \\
\hline Monopole mode & 1.250 & 1.254 & 22.95 & 22.74 & 12.9 & 12.9 \\
\hline Dipole mode & 1.719 & 1.725 & 50.96 & 48.13 & 0.27 & 1.15 \\
\hline
\end{tabular}

Due to the finite tolerances in machining, welding and mounting, some small distortions of cavity symmetry are generated. The dipole mode orthogonal polarizations show slightly different eigenfrequencies; the relative difference is, 
however, less than 2 per 1000 . Furthermore, the perfect polarization orthogonality is lost. A displacement of the beam in the ' $x$ ' direction gives not only a reading in that direction but also a non zero reading in the orthogonal direction ' $y$ '. This asymmetry is called cross talk.

Cross-talk isolation measurements are performed on the re-entrant cavity with a network analyzer. The transmission is first measured between two opposite antennas, the two other antennas are terminated with a $50 \Omega$ load. In this configuration, the monopole and dipole modes are excited as displayed in the Fig. 4. At the dipole frequency $(1.72 \mathrm{GHz})$, the transmission level is $-0.8 \mathrm{~dB}$.

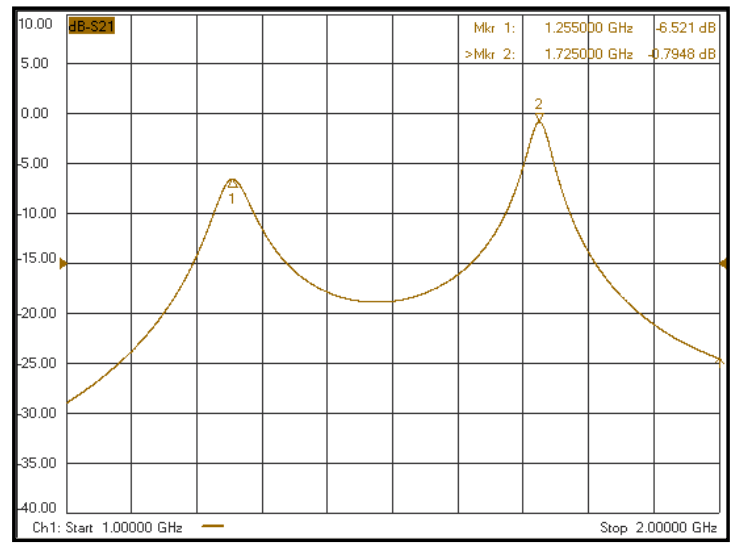

FIGURE 4. Monopole and dipole transmission measured by the network analyzer

For the second configuration, the signal transmission is measured between antennas positioned at $90^{\circ}$ through a $180^{\circ}$ hybrid coupler as shown in Fig. 5. The corresponding transmissions are displayed in Fig. 6. From those measurements, the cross-talk isolation value can be estimated about $41 \pm 1 \mathrm{~dB}$.

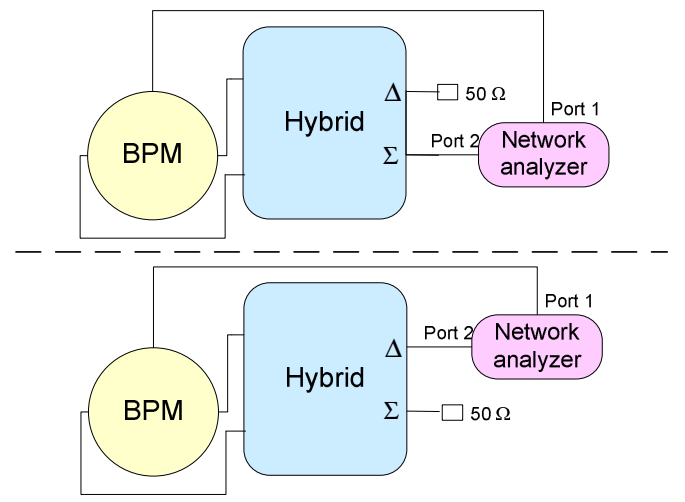

Figure 5. Representation of the cross talk measurement.

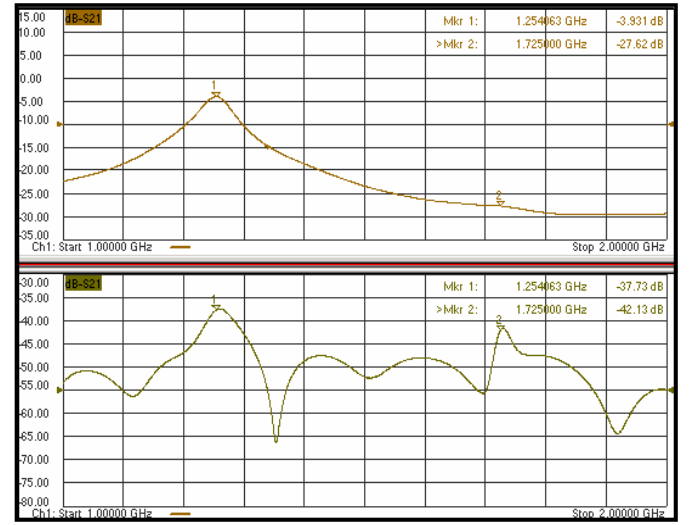

Figure 6. Signal coupled between two antennas positioned at $90^{\circ}$.

\section{RF Signal Processing Electronics}

The signal processing uses a single stage downconversion to obtain $\Delta / \Sigma$ and is shown in Fig. 7. It is composed of standard RF components: hybrid couplers, phase 
shifters, filters, isolators, mixers and amplifiers. The lowpass filter after the mixer has a bandwidth about $50 \mathrm{MHz}$, which determines the lowest bandwidth in the signal processing. The electronics is located outside the tunnel.

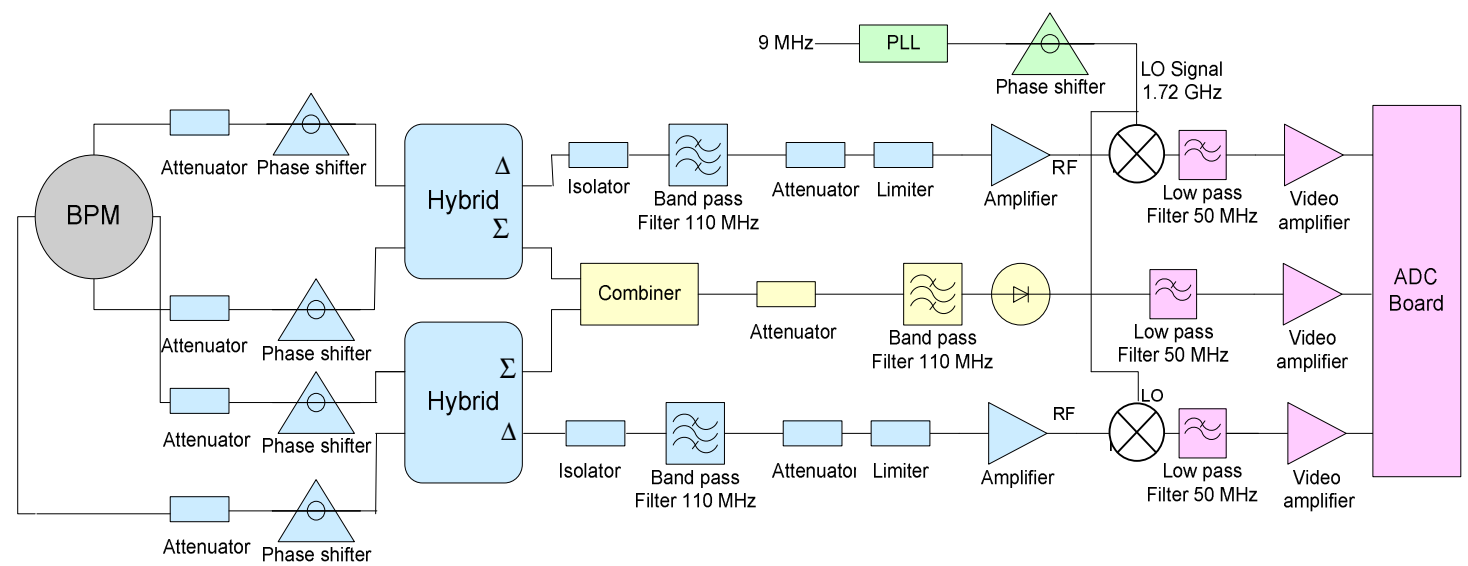

FIGURE 7: RF signal processing electronics

The hybrid coupler is connected to each pair of opposite antennas. The rejection of the monopole mode, on the $\Delta$ channel, proceeds in three steps:

- a mode symmetry based rejection with a hybrid coupler. Its isolation is higher than $25 \mathrm{~dB}$ in the range of 1 to $2 \mathrm{GHz}$. A local enhancement of the isolation to about $50 \mathrm{~dB}$ at the frequency of the dipole mode can be obtained with additional external phase shifters and attenuators.

- a frequency domain rejection with a band pass filter centered at the dipole mode frequency. Its bandwidth of $110 \mathrm{MHz}$ also provides a noise reduction.

- a synchronous detection of the dipole mode signal. A $9 \mathrm{MHz}$ reference signal is given by the linac control system and is combined with a PLL to generate a local oscillator signal (LO) for the mixers at the dipole mode frequency. Phase shifters are used to adjust the LO and RF signals in phase.

\section{ESTIMATION OF THE PERFORMANCES FOR DIFFERENT BPM SYSTEMS}

\section{Position Resolution and Offset}

The position resolution is the minimum position difference than can be statistically resolved. It is an RMS value related to the signal to noise ratio in the system. For the estimation, only the thermal noise, the noise figure and the noise generated in amplifiers are taken into account. The thermal noise of a system is given by the following equation:

$$
P_{t h}=k_{b} * T * B W
$$

where $\mathrm{k}_{\mathrm{b}}$ is Boltzmann's constant $\left(1.38^{*} 10^{-23} \mathrm{~J} / \mathrm{K}\right)$, BW is defined by the bandwidth of the signal processing channel in Hertz, and $\mathrm{T}$ is the room temperature in Kelvin. 
The noise level present at the output of the cavity BPMs is amplified by the devices which compose the signal processing. To calculate the noise level, the following equation is used:

$$
P_{n}=N F^{*} G^{*} P_{t h}
$$

where NF is the total noise figure of the signal processing channel, $G$ is the gain of the signal processing and $\mathrm{P}_{\text {th }}$ is the thermal noise. The total noise introduced into the system by the electronics can be evaluated by the noise figure in a cascaded system given by the following formula:

$$
N F=F_{1}+\frac{F_{2}-1}{G_{1}}+\frac{F_{3}-1}{G_{1} * G_{2}}+\ldots
$$

where NF is the total noise factor of the signal processing, $F_{i}$ and $G_{i}$ are respectively the noise factor and the gain of component $i$.

To assess the performance of the system, a model (cavity+signal processing) is elaborated with Mathcad. The RF re-entrant cavity model is a resonant RLC circuit; the single bunch response of the cavity depends on frequency and external coupling of the modes (monopole and dipole). The transfer functions of different elements (cables, hybrid couplers, filters, amplifier mixer) composing the signal processing are determined by the $\mathrm{S}$ parameters measured with a network analyzer. Then, those transfer functions are used and combined to simulate the BPM system (RF cavity+signal processing). The transfer function of cables takes into account the effect of attenuation and dispersion.

The system offset is determined by the contamination of the monopole mode, the rejection of which is never totally perfect in practice. The following tables 3 and 4 compare three systems regarding the offset, the resolution, noise level and signal level. The first system corresponds to the BPM installed in ACC1 (9ACC1) on TTF2. The second system is identical to 9ACC1 except the Bessel filter bandwidth, which is increased to $110 \mathrm{MHz}$. The gain was adjusted to have an RF signal level around $0 \mathrm{dBm}$ on the $\Delta$ channel for $10 \mu \mathrm{m}$ beam offset. The third system (NBPM) is described in the previous section. Its gain is also adjusted to have the RF signal level around $0 \mathrm{dBm}$ for the same offset. For the two last systems, as the bandpass filter bandwidth $(110 \mathrm{MHz})$ is larger than the one of the lowpass filter $(50 \mathrm{MHz})$, the thermal noise power is defined by the lowpass filter bandwidth, i.e. BW is equal to $50 \mathrm{MHz}$ in equation 1 . A room temperature prototype of the NBPM system is ready for warm tests with beam on TTF2.

TABLE 3. Signal and noise level for reentrant BPM systems

\begin{tabular}{|l|c|c|c|}
\hline Systems & $\begin{array}{c}\text { Signal on } \boldsymbol{\Delta} \text { channel with } \\
\mathbf{1 0} \boldsymbol{\mu m} \text { beam offset } \mathbf{( d B m})\end{array}$ & $\begin{array}{c}\text { Total noise } \\
(\mathbf{d B m})\end{array}$ & $\begin{array}{c}\text { Thermal noise } \\
(\mathbf{d B m})\end{array}$ \\
\hline 9ACC1 with 8 MHz Bessel BPF & -79 & -57.2 & -104.9 \\
\hline 9ACC1 with 110 MHz Bessel BPF & -5.8 & -28.4 & -96.9 \\
\hline NBPM & -9.3 & -50.8 & -96.9 \\
\hline
\end{tabular}


TABLE 4. Position resolution and offset of reentrant BPM systems

\begin{tabular}{|l|c|c|}
\hline Systems & Simulated resolution $(\boldsymbol{\mu m})$ & Offset $(\boldsymbol{\mu m})$ \\
\hline 9ACC1 with 8 MHz Bessel BPF & 6.0 & 6.8 \\
\hline 9ACC1 with 110 MHz Bessel BPF & 1.4 & 6.0 \\
\hline NBPM & 0.12 & 1.4 \\
\hline
\end{tabular}

If one assume that the other noise sources related to the environment, which are not accounted for in the simulation, have the same white noise characteristics as the thermal noise, a comparison of the $6 \mu \mathrm{m}$ simulated resolution to the $30 \mu \mathrm{m}$ experimental resolution for the BPM 9ACC1 with $8 \mathrm{MHz}$ bandwidth would give an estimation of the "real" noise power at the input of the signal processing channels. This "real" noise power may be applied to the NBPM system in the simulation to predict a resolution around $0.2 \mu \mathrm{m}$ instead of $0.12 \mu \mathrm{m}$ while only the thermal noise power was considered.

\section{Time Resolution}

One of the most important parameters for a BPM is the time resolution, which is usually identified to the damping time. For bunch to bunch measurements, the time resolution has to be smaller than the distance between the bunches. The damping time is given by the following formula [4]:

$$
\tau=\frac{1}{\pi * B W}
$$

where $\mathrm{BW}$ is the bandwidth, defined by the relation:

$$
B W=\frac{f_{d}}{Q_{l d}} .
$$

with $f_{d}$ the frequency and $Q_{l d}$ the loaded quality factor for the dipole mode. The time resolution is therefore around $9.5 \mathrm{~ns}$ for the new re-entrant BPM. It is lower than the separation between bunches on TTF2 (110ns); bunch to bunch measurement is therefore possible.

In practice, the rising time to $95 \%$ of a cavity response signal is $3 \tau$. For bunch to bunch measurements, the time resolution has to be smaller than the distance between bunches $\Delta \mathrm{T}$, the system has to verify the following equation:

$$
6 \tau \leq \Delta T
$$

To evaluate the time resolution of the BPM systems (cavity+electronics), the model is used to simulate the output signal after the synchronous detection. The time resolution is therefore defined by the time interval at $5 \%$ of the peak voltage from the baseline. Table 5 proves that the BPM system installed in ACC1 could not perform bunch to bunch measurements and the need to change the filter bandwidth.

TABLE 5. Time resolution of reentrant BPM systems.

\begin{tabular}{|l|c|c|}
\hline Systems & $\begin{array}{c}\text { Damping time (ns) } \\
\text { cavity only }\end{array}$ & $\begin{array}{c}\text { Time resolution (ns) } \\
\text { cavity+electronics }\end{array}$ \\
\hline 9ACC1 with 8 MHz Bessel BPF & 0.63 & 204 \\
\hline 9ACC1 with 110 MHz Bessel BPF & 0.63 & 20 \\
\hline NBPM & 9.4 & 40 \\
\hline
\end{tabular}




\section{CONCLUSION}

This new system is a compromise to have a high resolution down to $1 \mu \mathrm{m}$ and to have the possibility to do bunch to bunch measurements for XFEL or ILC. The simulation results for the new BPM system are very encouraging. The time resolution is lower than $50 \mathrm{~ns}$ and the predictable resolution is around $0.2 \mu \mathrm{m}$. This BPM is designed to be used in a clean environment, at cryogenic or room temperature. Its main features are the small size of the RF cavity, a large aperture $(78 \mathrm{~mm})$ and an excellent linearity. Summer 2006, the first tests with the beam will start at room temperature. This BPM appears as a good candidate for being installed in the XFEL and ILC cryomodules.

\section{ACKNOWLEDGMENTS}

We acknowledge the support of the European Community-Research Infrastructure Activity under the FP6 "Structuring the European Research Area" programme (CARE, contract number RII3-CT-2003-506395).

\section{REFERENCES}

1. R.Bossart, "High precision beam position monitor using a re-entrant coaxial cavity" Processings of LINAC94, KEK, (1994).

2. C.Magne, et al., "High resolution BPM for the future linear colliders", LINAC98 Conference, 1998

3. C Magne, et al, "Reentrant cavity BPM for linear colliders", BIW2000R.

4. V.Sargsyan, "Cavity Beam Position Monitor for the Tesla-cryomodule. Cross-Talk minimization”, Berlin 2003 